\title{
Information Contents of a Signal at Repeated Positioning Measurements of the Coordinate Measuring Machine (CMM) by Laser Interferometer
}

\author{
Tomáš Stejskal ${ }^{1}$, Tatiana Kelemenová ${ }^{2}$, Miroslav Dovica ${ }^{2}$, Peter Demeč ${ }^{1}$, Miroslav Štofa ${ }^{1}$ \\ ${ }^{I}$ Technical University of Košice, Faculty of Mechanical Engineering, Department of Production Technique, Letná 9 , \\ 04002 Košice Slovakia, tomas.stejskal@tuke.sk \\ ${ }^{2}$ Technical University of Košice, Faculty of Mechanical Engineering, Department of Biomedical Engineering and \\ Measurement, Letná 9, 04002 Košice Slovakia
}

\begin{abstract}
The input of this paper lies in displaying possibilities how to determine the condition of a coordinate measuring machine (CMM) based on a large number of repeated measurements. The number of repeated measurements exceeds common requirements for determining positioning accuracy. The total offset in the accuracy of spatial positioning consists of partial inaccuracies of individual axes. 6 basic errors may be defined at each axis. In a triaxial set, that translates into 18 errors, to which an offset from the perpendicularity between the axial pairs must be added. Therefore, the combined number of errors in a single position is 21 . These errors are systemic and stem from the machine's geometry. In addition, there are accidental errors to account for as well. Accidental errors can be attributed to vibrations, mass inertness, passive resistance, and in part to fluctuations in temperature. A peculiar set of systemic errors are time-varying errors. The nature of those errors may be reversible, for instance if they result from influence of temperature or elastic deformation. They can be also irreversible, for example as a result of wear and tear or line clogging, due to loosened connection or permanent deformation of a part post collision. A demonstration of thermal equalizing of the machine's parts may also be observed in case of failure to adhere to a sufficient time interval from the moment the air-conditioning is turned on. Repeated measurements done on a selected axis with linear interferometer can provide complex information on the CMM condition and also on the machine's interaction with the given technical environment.
\end{abstract}

Keywords: Laser interferometer, CMM, machine's condition.

\section{INTRODUCTION}

Repeated measurements of the CMM portal position in one axis only provide not only information on some errors but also complex information on the machine's interaction with its environment and on condition of its functional elements. In this respect, it is desirable to realize that a metrologist needs not only information on the machine's calibration, but also on changes in the machine's condition, which might result in a subsequent measurement error or reduced operability of the measurement process. It is exactly this important information that is encoded in the measured signals from the CMM axial positioning.

Utilization of the laser interferometer and CMM combination is also suitable for calibration of length measuring devices with lower order of accuracy than a laser. It is an indirect measuring method, where CMM only delivers measuring movements of the reflector against the measured object, but the length values are provided for by the laser interferometer [1]. A disadvantage of this method is that the reflector must be located in the holder of the sensor head while the laser beam must not be interrupted. This translates into significant movement restrictions of the measuring process.

Due to that, the CMM development steers in the direction of optical systems of measurement. [2]. Optical systems also allow for special measuring tasks. For example, dimensional measurement of micro caviti profile with a special optical probe [3].

Another development has taken place in increasing the accuracy of laser measuring systems for measuring the accuracy of CMM positioning. Best results have been achieved following the principle of an optical-comb pulsed interferometer. Then, a compact absolute positionmeasuring system is realized for practical non-contact use with high accuracy of measurement [4].

Optical CMM systems allow for new tasks to be performed. For example, large objects can be measured, such as large gears with diameters up to $1000 \mathrm{~mm}$. This development also envisages creation of new standards of measurement [5]. 
Indirect methods of condition evaluation are applied in various ways. For example, for tuning at low resonance, the receiver coil is tuned by utilizing a noise signal from the device [6]. By analogy, this study also uses indirect evaluation of accuracy, since the signal for measuring length by laser interferometer in one axis of CMM portal positioning shows its overall working condition. The entire setup for measuring portal position by laser interferometer could be also used for indirect measuring of size of an object placed on the CMM table. The condition is to determine uncertainty of measurement in the given axis. The uncertainty of measurement is contributed to by the entire kinetic chain from the foot of the portal to the measuring probe. The issue of simplified determination of uncertainty of measurement is discussed in papers [7], [8]. However, this is not a suitable method for the case at hand. The method of evaluating the values taken by measurements may yield better results, also taking into account their uncertainty, i.e., this should not be limited to mere statistical evaluation of measured points at repeated measurements. [9].

An unequivocal verification of the machine's volumetric accuracy makes sense only when the aim is correction of the machine's error map. In this way, the influence of some geometrical systemic errors should be minimized to the least tolerable value if not eliminated entirely. This is a demanding measuring of many points in space, while in theory, each point may display 21 possible errors. Standards allow for simplified procedures in measuring volumetric accuracy, where some errors are ignored depending on the method of measurement applied. The result of complex measuring of volumetric accuracy itself is relevant only if the machine's error map may be corrected in its wake. However, this is an issue for CMM servicing companies to tackle, or it is the know-how domain of the CMM manufacturers. Verification measurements of volumetric accuracy are simplified and they provide only information that, over a specified period of time, default inaccuracy limits of CMM were not exceeded. It is good to bear in mind that the error map is a form of flexible automatization and it is immediately connected with the machine's hardware. In mass production, the kinematics and dynamics of machines is ensured via hard (innumerical) automatization even today. Final accuracy may be achieved either through exact production of a surface without correction of systemic offsets or a less exact production of a surface, to which correction of systemic offsets that eliminate manufacturing inaccuracy is applied. Thus, we do not speak of software in the sense of a control program; rather, we speak of an integral part of the hardware.

Information retrieved in course of special measuring procedure proposed in this paper aim at optimization of measuring conditions and periodic monitoring of the machine's condition. Early detection of deficiencies resulting from such measurements saves future costs that might result from lack of sufficient information on real condition of a CMM. They also indirectly correlate with possibilities of increased measurement accuracy in special cases based on real requirements. From this aspect, the metrologist needs to know the machine's behavior as influenced by ambient temperature conditions and the time of measurement, translating into final accuracy.

\section{CONCEPT OF MEASURE TAKING IN A SINGLE AXIS}

The measures were taken at a CMM with a coordinate measuring head. This machine type compensates for dynamic errors of measurement significantly better than a swinging measuring head. At repeated measurements taken only at a single point, influence of geometrical errors is eliminated and only an accidental component of an error is displayed, recorded by the laser inteferometer in real time.

Repeated measurements taken at multiple points without probe deployment onto the measured object provide good information on temperature stability of the machine and its environment. At the same time, information is received on how the driver engine is positioned in the measured axis and on the correctness of mechanical transfer. The errors mentioned above do not impact the final accuracy of measurement as they are eliminated by accuracy of the ruler and its single-point alignment in the given axis. If critical limit is exceeded, said errors may manifest themselves as measurement inaccuracy.

Measuring a single axis also has an economic upside, because its preparation and the alignment of measuring components is not difficult. In addition, it does not have to depend on a service provider. In certain sense, due to great number of measurements, the activity becomes time consuming. However, information on time variability is valued nevertheless.

The concept of measure taking in a single axis is also justified from the calibration aspect, or from the point of view of verification of the machine's calibration. If it is assumed that the machine meets all required accuracy conditions post calibration, then this condition can only deteriorate with time. Degree of deterioration may be detected indirectly, exactly by applying the concept of measure taking in a single axis. Therefore, it is good to preserve records of measurements taken immediately post machine calibration. Such record can then serve as a reference value against which measurements taken after some time has lapsed can be compared. The machine's deteriorated condition will be thus displayed via significant offsets from the reference value measurement.

\section{CONFIGURATION OF MEASUREMENTS TAKEN BY THE LASER INTERFEROMETER}

Individual measurement configurations served the purpose of detecting main effects influencing the course of measure taking.

\section{First measure taking configuration:}

Laser interferometer is aligned to measure axis $\mathrm{Y}$, which is usually the most accessible part of the machine. The measures of measured points $25 \mathrm{~mm}$ apart were taken in five positions over $100 \mathrm{~mm}$. The room was air-conditioned to $\left(20{ }^{\circ} \mathrm{C}\right)$. The measures were taken at bilateral assumption of programed positions. The interferometer's reflector was 
magnetically attached to the carriage. Interferometer was magnetically attached to a steel platform placed in an extreme position of a granite slab (Fig.1.).

Measures were taken in automated mode. The number of repeated cycles was 300 in each direction. Total measuring time was 4 hours and 11 minutes.

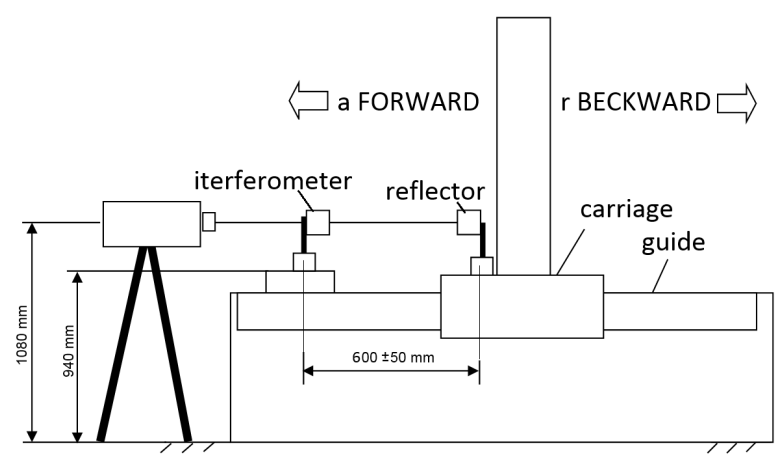

Fig.1. First and third configuration layout of measurement by laser interferometer.

\section{Second measure taking configuration:}

Measure taking modification meant turning off the airconditioning, while the CMM portal was not performing any movements throughtout the course of measure taking. Thus, a change in distance between the reflector and the interferometer due to change in the ambient temperature was captured. The distance between the interferometer and the reflector was $470 \mathrm{~mm}$. It is necessary to consider a somewhat greater share of a larger distance in thermal deformations since the interferometer was placed on a steel platform and the reflector on the carriage (Fig.2.).

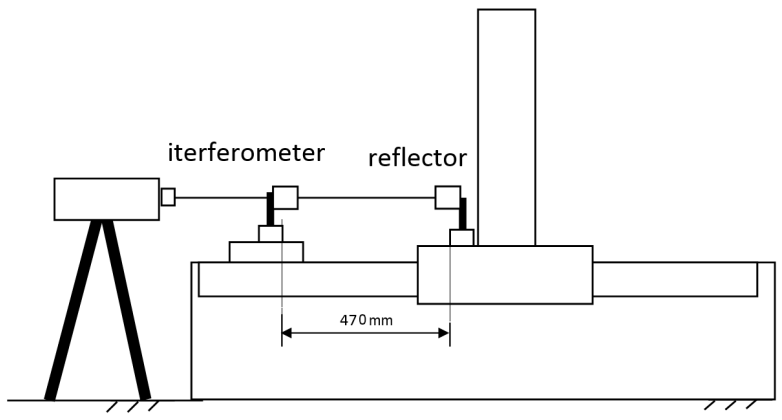

Fig.2. Second configuration layout of measurement with inactive portal.

\section{Third measure taking configuration:}

The modification meant repeated deployment of the measuring head onto the measured object. The ruler recording was done at the moment the head touched the object. Simultaneously, the portal's position in the $\mathrm{Y}$ axis was measured by the interferometer.

\section{INTERPRETATION OF MEASURED RESULTS}

The analysis of measured values provided an answer as to the impact of the ambient temperature and effectiveness of air-conditioning in given conditions. A standard deviation from the size of accidental error of the measuring head deployment onto the measured object was assessed. The condition of the $\mathrm{Y}$ axis slide was evaluated.

\section{Impact of the change in ambient temperature}

The change in ambient temperature may be interpreted by comparing outcomes of the first and the second configuration of measure taking. Air-conditioned temperature actually dynamically keeps the CMM ambient temperature stable. This dynamics will also demonstrate itself in the results of repeated measurements in a single axis. Fig.3. shows significant waves cyclically reemerging about every 13 minutes at position assumption in the direction. This is a result of regulated air-conditioning. In spite of that, after approximately 150 minutes into the measurement, an emerging trend may be observed, caused by thermal deformations of the measured set. The figure shows the position of two adjacent measured points. There is an obvious correlation between these two points, which proves the trend shows a systemic change.

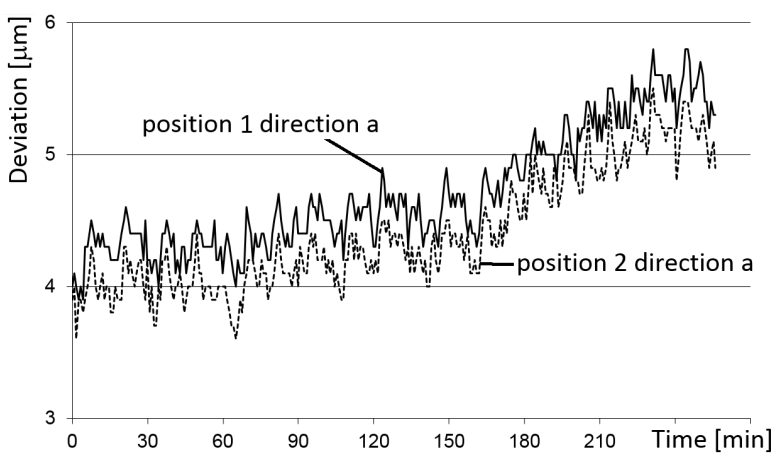

Fig.3. First configuration layout of measurement. Trend comparison between two adjacent positions.

A proof of the fact that the distance dynamically changes even with a small change in temperature is the reading from the second measure taking configuration. The intensity of the change in size at the beginning of measurement is markedly greater than at the end of measurement. (Fig.4.).

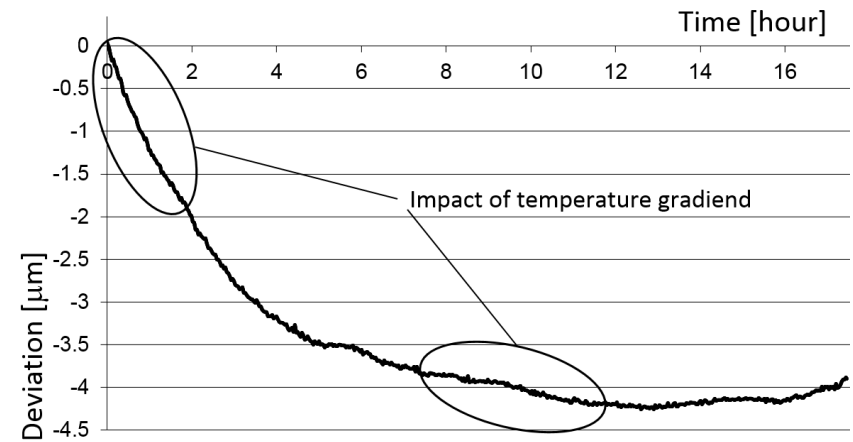

Fig.4. Second configuration layout of measurement. Change of distance between two points in the gradual change of the ambient temperature. 
Gradient of thermal change is steepest over a two-hour interval. After 10 hours, thermal stabilization can be observed, while the ambient temperature rose by $5{ }^{\circ} \mathrm{C}$. This thermal balancing was examined at the distance of 50 centimeters, with metallic set components having a share in the change (for instance the foot of the portal). The difference between the ambient temperature and the airconditioned temperature markedly impacts thermal dynamics of the machine.

The degree of an accidental error occurring at assumption of the measured position may be estimated by the third measure taking configuration. This is a complicated issue because the portal's position towards the ruler is compensated by measuring head with its own system of measurement. Only the portal ruler values and the interferometer values were available. Unfortunately, no measuring head values were obtained to compensate the result with. In spite of that the obtained values evidence dynamics of position assumption. Were it not necessary to compensate the assumption of the measuring position, in theory, there should be high correlation of deviations measured by the ruler and the interferometer.

The value of measured correlation is 0.246 . It is a rather small correlation. A glimpse at the measurement results could, after all, justify estimating a certain tendency for change of the mean value (Fig.5.).

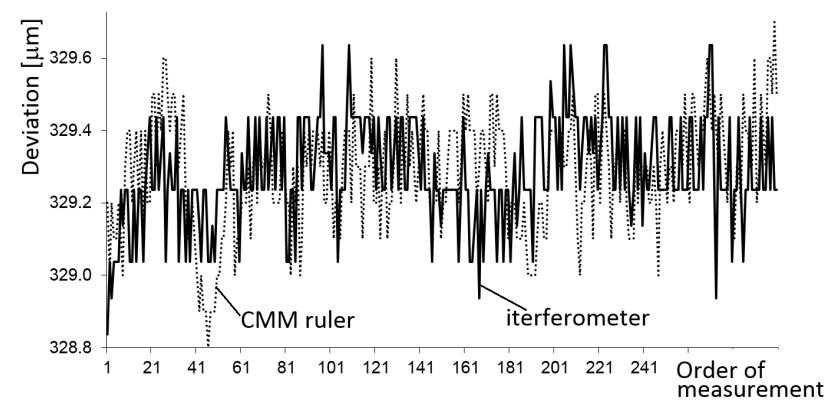

Fig.5. Comparison of change in the value of the ruler and the interferometer at the moment they touched the measured object at repeated measurements.

\section{Evaluation of normality in the measured data set and the uncertainty of measurement}

It is appropriate to display the measured values as multiplicity histograms. The number of class intervals $k_{\text {hist }}$ can be determined by application of the Sturgess rule [10]:

$$
k_{\text {hist }} \doteq 1+\log _{2} n_{M}
$$

where the number of measured data $n_{M}=300$ is the number of class intervals 10. Limit values of class intervals are determined by division of the range of values of the measured set into 10 parts, from which it is then possible to create a histogram (Fig.6., Fig.7.). The latter offers a picture of how the measured data are spread.

Depicted courses (Fig.6., Fig.7.) give rise to an $H_{0}$ hypothesis, which says the measured values are likely to follow the normal spread law. This hypothesis may be conveniently verified by good match tests (normality test) [11], [12]. There are several types of normality tests, with each of them having its own specifics, suitable for certain data sets. A test with the greatest strength (i.e., to detect and reject normality) out of all tests in use is the Shapiro-Wilk test. Where certain values reemerge repeatedly, the Kolmogorov-Smirnov good match test is recommended [13].

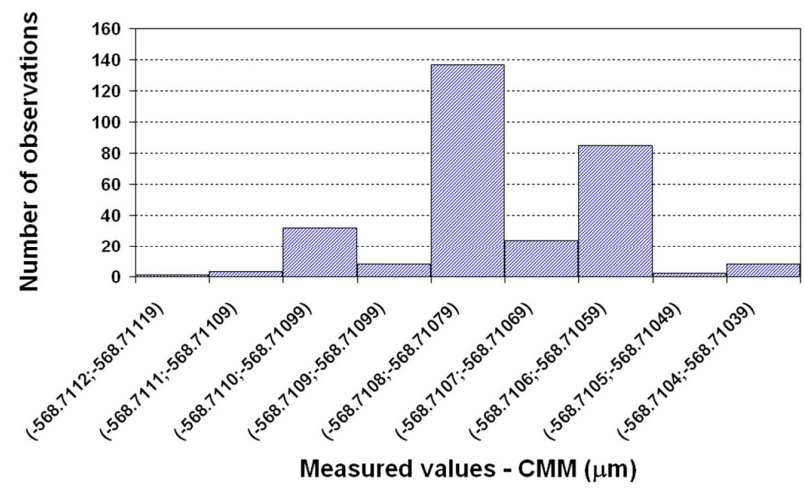

Fig.6. Histogram of the values measured by the coordinate measuring machine CMM.

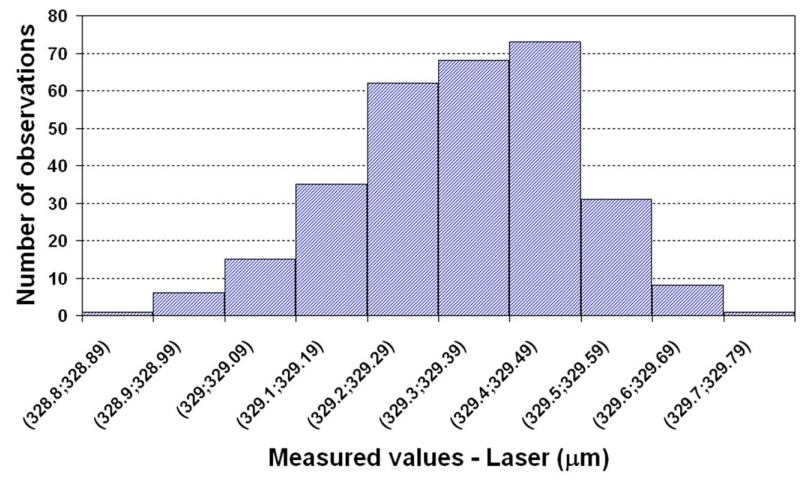

Fig.7. Histogram of the values measured by the laser interferometer.

Three types of normality tests were used to assess the measured data set normality:

- Kolmogorov-Smirnov test (K-S test),

- K-S, Lilliefors test,

- Shapiro-Wilks' W test.

Kolmogorov-Smirnov test (K-S test): If the tested property $D_{\max }$ is greater than the critical value $D_{a}$, then the zero normality hypothesis is rejected. With this test, $k_{K S}$ classes of tested set are created together with their theoretical normal distribution, divided into an equal number of classes. For each class of the tested set the multiplicity $n_{l i}$ is calculated together with the multiplicity $n_{2 i}$ of each class of theoretical normal distribution. The next step is determination of cumulative class multiplicity of the tested set:

$$
N_{1 i}=\sum_{j=1}^{i} n_{1 j}
$$


and of cumulative class multiplicity of the set according to theoretical normal distribution:

$$
N_{2 i}=\sum_{j=1}^{i} n_{2 j}
$$

Tested property then equals:

$$
D_{1}=\frac{1}{n_{M}} \cdot \max _{i}\left|N_{1 i}-N_{2 i}\right|
$$

The tested property $D_{1}$ is then compared to the critical value $D_{\alpha}$. At the significance level of 0.05 the critical value is (for $n_{M}>35$ ):

$$
D_{\alpha}=\frac{1.36}{\sqrt{n_{M}}}
$$

If the tested property limit is exceeded, the zero normality hypothesis $H_{0}$ is rejected. The Kolmogorov - Smirnov test results (Table 1.) rejected the $H_{0}$ normality hypothesis of the measured data in both tested sets.

Table 1. Kolmogorov-Smirnov test results.

\begin{tabular}{|l|l|l|l|l|}
\hline $\begin{array}{l}\text { Kolmogorov- } \\
\text { Smirnov Test }\end{array}$ & $n_{M}$ & $D_{l}$ & $D_{a}$ & Result \\
\hline Laser & 300 & 0.143458 & 0.0785 & $\begin{array}{l}D 1>D a \\
\rightarrow H_{0} \\
\text { rejected }\end{array}$ \\
\hline CMM & 300 & 0.236824 & 0.0785 & $\begin{array}{l}D 1>D a \\
\rightarrow H_{0} \\
\text { rejected }\end{array}$ \\
\hline
\end{tabular}

K-S Lilliefors test: The applied K-S test was a modified one. The basis for calculation of the tested property is the Kolmogorov-Smirnov test and the critical value under consideration is that according to the Lilliefors probability applied to evaluation of test results. The K-S Lilliefors test results (Table 2.) rejected the $H_{0}$ normality hypothesis in both tested data sets.

Table 2. K-S Lilliefors test results.

\begin{tabular}{|l|l|l|l|l|}
\hline $\begin{array}{l}\text { K-S, Lilliefors } \\
\text { test }\end{array}$ & $n_{M}$ & $D_{l}$ & $D_{\alpha}$ & Result \\
\hline Laser & 300 & 0.143458 & 0.045 & $\begin{array}{l}D 1>D a \\
\rightarrow H_{0} \\
\text { rejected }\end{array}$ \\
\hline CMM & 300 & 0.236824 & 0.045 & $\begin{array}{l}D 1>D a \\
\rightarrow H_{0} \\
\text { rejected }\end{array}$ \\
\hline
\end{tabular}

Shapiro-Wilks W test is the most preferred normality test, thanks to its good strength in comparison to a wide range of other normality tests.

Tested property is expressed by the following relation:

$$
W=\frac{\left(\sum_{i=1}^{n} a_{i} x_{(i)}\right)^{2}}{\sum_{i=1}^{n}\left(x_{i}-\bar{x}\right)^{2}}
$$

where:

$x_{i}$ is the measured value of the tested set; $\bar{x}$ is an arithmetic mean; $a_{i}$ is expressed by the relation:

$$
\left(a_{1}, \ldots, a_{n}\right)=\frac{\mathbf{m}^{\mathrm{T}} \mathbf{V}^{-1}}{\left(\mathbf{m}^{\mathrm{T}} \mathbf{V}^{-\mathbf{1}} \mathbf{V}^{-1} \mathbf{m}\right)^{\mathbf{1} / \mathbf{2}}}
$$

where:

$\mathbf{m}=\left(m_{1}, \ldots, m_{n}\right)^{T}$ are expected values of distribution function of normal spread; $\boldsymbol{V}$ - is a co-variable matrix.

Zero normality hypotheses is rejected if the tested property $W$ is too low. The Shapiro-Wilks test results (Table 3.) rejected the $H_{0}$ normality hypothesis in both tested data sets.

Table 3. Shapiro-Wilks test results.

\begin{tabular}{|l|l|l|l|l|}
\hline $\begin{array}{l}\text { Shapiro-Wilks } \\
\text { test }\end{array}$ & $n_{M}$ & $W$ & $W^{\prime}$ & Result \\
\hline Laser & 300 & 0.948682 & 0.9905 & $\begin{array}{l}\mathrm{W}<\mathrm{W}^{\prime} \\
\rightarrow H_{0} \\
\text { rejected }\end{array}$ \\
\hline CMM & 300 & 0.879276 & 0.9905 & $\begin{array}{l}\mathrm{W}<\mathrm{W}^{\prime} \\
\rightarrow H_{0} \\
\text { rejected }\end{array}$ \\
\hline
\end{tabular}

All tests that were performed with respect to hypothesis of normal distribution in the basic set rejected the zero hypothesis, which assumes that distribution with an unknown distribution function $F(x)$ matches the distribution with a known distribution function $F_{0}(x)$ in normal theoretical spread [14]. Therefore, the same probability of occurrence of any deviation in the interval of measured values can be expected. No information on distribution of probability of deviation occurrence is available to accordingly assess the measurement, thus there is no reason to prefer any certain deviation. It holds for rectangular uniform distribution that the probability of occurrence of each value in the basic set interval in the course of measurement is equally likely.

Measurement conditions enable us to set a standard uncertainty of measurement solely by application of the B method, setting the maximum error allowed pursuant to data from an interferometer manufacturer (Maximum permission error $\left.M P E_{L A S E R}= \pm 0.5 \mu \mathrm{m}\right)$. Thus, uniform distribution at $95 \%$ probability can be translated into a coverage factor of $k_{\text {LASER }}=\sqrt{3}$. In such case, the uncertainty of measurement with a laser interferometer can be expressed through the B method by the following formula:

$$
u_{B L A S E R}=\frac{M P E_{L A S E R}}{k_{L A S E R}}=0.29 \mu \mathrm{m} .
$$


By analogy, for measuring with a coordinate measuring machine (CMM), a manufacturer specifies the maximum permission error $M P E_{C M M}= \pm 1.8+\mathrm{L} / 300 \mu \mathrm{m}$.

Thus, the uncertainty of a particular measurement taken in a given point by the coordinate measuring machine can be expressed by the B method in the following formula:

$$
u_{B C M M}=\frac{M P E_{C M M}}{k_{C M M}}=1.23 \mu \mathrm{m} .
$$

Uniform rather than normal distribution of values from repeated measurements confirms high quality of CMM positioning dynamics. No value dispersion occurs in an ideal case. Comparison of histograms of data measured by the CMM and laser, (Fig.6.) and (Fig.7.), shows that the laser displays a greater range of class intervals. This fact evidences thermal dilatations that naturally occur in the course of measurement between the axis $\mathrm{Y}$ ruler, the reflector and the interferometer.

\section{Actuator condition}

The most significantly demonstrated condition at repeated measurements is that of the actuator. This is evident from the very first measurement configuration, when results of position assumption in both directions are compared (Fig.8). As is apparent, the values in $\mathbf{r}$ direction are significantly more dispersed than in the a direction, which is likely related to insufficient perpendicularity of the axis of friction transmission against the machine's grid axis. This is not a critical shortcoming from the accuracy of measurement viewpoint, because this value dispersion is reliably compensated by the axial ruler. However, deterioration in condition over time may by later demonstrated by the actuator's more intense wear and tear, with severity of damage intensely increasing.

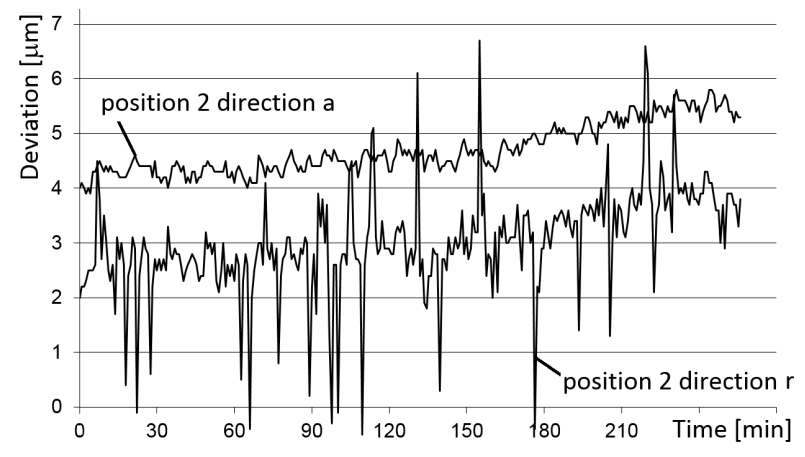

Fig.8. Course of repeated measurements at the same position, but in various directions $(a, r)$.

An advantage of this type of error monitoring is, among other things, the fact that reviews by interferometer are no longer necessary. Thus, obtained transmission anomaly is captured in the reading of the log of position measuring against the ruler at great number of repeated measurement takings (Fig.9.). All axes of the machine can be reviewed in this manner. Occurrence of fat tails after repeated measurements is exponential in nature, with mean value of 5.36. The number of sufficient repeated measurements can be reduced by estimation to 16 (three times the standard deviation). This follows from the analysis of average occurrence of fat tail changes.

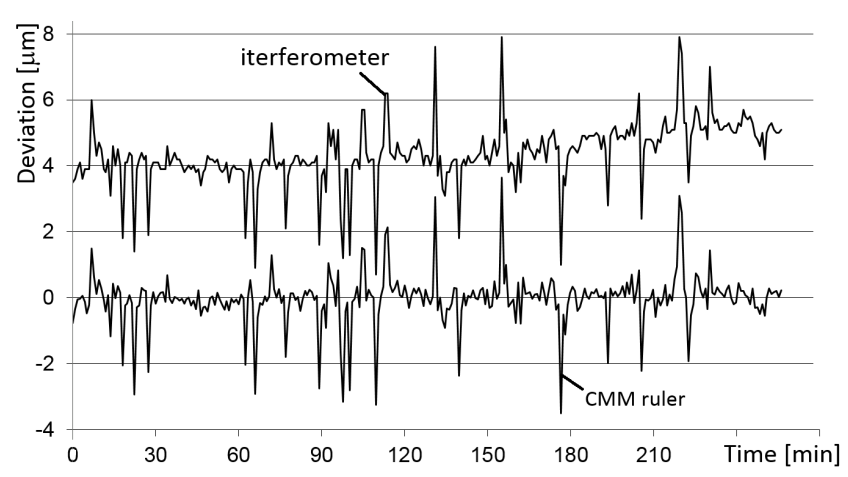

Fig.9. Comparison of deviations measured by interferometer against the ruler log reading ( $\mathrm{r}$ direction).

\section{CONCLUSION}

The proposed procedure of measurement in a single CMM axis relates only indirectly to reviewing precision of the machine. Greater emphasis is given to monitoring the machine's condition. In such case, air-conditioning and actuator defects are well detected. This is an important piece of information that can significantly save the overall operating costs of exposed devices. Repeated measurements are indeed time consuming, on the other hand, they can provide complex information about the condition. Likewise, tt has been proven that low number of repeated measurements might lead to incorrect evaluation conclusions. Fat tail changes resulting from transmission error were of accidental nature. They might not be detected at all in less voluminous series of measurements. Longer measurement taking time detects also the machine's propensity to thermal changes, which is also an important piece of information.

\section{ACKNOWLEDGMENT}

We owe our gratitude to Mr. Jozef Kováč, MSc. for his expert help with measurements and processing of their results.

The paper was published under the grant project OPVaV2009/2.1/03-SORO framework. ITMS code: 26220120060 „Center for research of technical, environmental and humanitarian risk management to ensure sustained development of production and products in mechanical engineering ".

Contribution was created under grant projects KEGA 039TUKE-4/2016, VEGA 1/0182/15, VEGA 1/0124/15 and APVV-15-0149 Research of new measuring methods of machine condition. 


\section{REFERENCES}

[1] Acko, B. (2007). Calibration of measuring instruments on a coordinate measuring machine. Advances in Production Engineering and Management, 2 (4), 12734.

[2] Larue, J.-F., Brown, D., Viala, M. (2015). How optical CMMs and 3D scanning will revolutionize the 3D metrology world. In Integrated Imaging and Vision Techniques for Industrial Inspection. Springer, 141176.

[3] Zhang, K., Zhang, Q., Lin, C.-D., Fan, K.-C. (2015). A visual non-contact focusing probe for the measurement of micro cavities. International Journal of Nanomanufacturing, 11 (3-4), 207-218.

[4] Sudatham, W., Matsumoto, H., Takahashi, S., Takamasu, K. (2015). Verification of the positioning accuracy of industrial coordinate measuring machine using optical-comb pulsed interferometer with a rough metal ball target. Precision Engineering, 41, 63-67.

[5] Acko, B., McCarthy, M., Haertig, F, Buchmeister, B. (2012). Standards for testing freeform measurement capability of optical and tactile coordinate measuring machines. Measurement Science and Technology, 23 (9), 094013.

[6] Andris, P., Dermek, T., Frollo, I. (2015). Simplified matching and tuning experimental receive coils for low-field NMR measurements. Measurement, 64, 2933.

[7] Kušnerová, M., Valíček, J., Harničárová, M., Hryniewicz, T., Rokosz, K., Palková, Z., Václavík, V., Řepka, M., Bendová, M. (2013). A Proposal for simplifying the method of evaluation of uncertainties in measurement result. Measurement Science Review, 13 (1), 1-6.

[8] Kureková, E., Halaj, M., Omachelová, M., Martišovitš, I. (2014). Theoretical positioning accuracy for serial and parallel kinematic structure. Measurement Science Review, 14 (5), 243-251.

[9] Palenčár, R., Halaj, M., Kureková, E. (2007). Evaluation of the positional deviation of numerically controlled axes. Measurement Science Review, 7 (1), 27-30.

[10] Sturges, H.A. (1926). The choice of a class interval. Journal of the American Statistical Association, 21 (153), 65-66.

[11] Meloun, M., Militký, J. (2004). Statistical analysis of experimental data, (in Czech). Prague, Czech Republic: Academia, 2004. ISBN 80-200-1254-0.

[12] Ostertagová, E. (2011). Applied statistics, (in Slovak), 1st Edition. Košice, Slovakia: Elfa Publishing. ISBN 978-80-8086-171-1.

[13] Rimarčík, M. (2007). Statistics for praxis, (in Slovak), 1st Edition. ISBN 978-80-969813-1-1.

[14] Palenčár, R., Ruiz, J.M., Janiga, I., Horníková, A. (2001). Statistical methods in metrological and testing laboratories, (in Slovak), Bratislava, Slovakia: Ing. Peter Juriga - Grafické štúdio. ISBN 80-968449-3-8.

Received May 25, 2016. Accepted October 17, 2016 